\title{
Initiation and chromospheric effects of a M1.0 class solar flare from high-resolution multi-wavelength observations
}

\author{
V. M. Sadykov ${ }^{1}$, A. G. Kosovichev ${ }^{1,2,3}$, \\ I. N. Sharykin ${ }^{4}$ I. V. Zimovets ${ }^{4}$ and S. Vargas Dominguez ${ }^{5}$ \\ ${ }^{1}$ Department of Physics, New Jersey Institute of Technology, Newark, NJ 07102, USA \\ email: vsadykov@njit.edu \\ ${ }^{2}$ NASA Ames Research Center, Moffett Field, CA 94035, USA \\ ${ }^{3}$ W.W. Hansen Experimental Physics Laboratory, Stanford University, \\ Stanford, CA 94305, USA \\ ${ }^{4}$ Space Research Institute (IKI) of Russian Academy of Sciences, Moscow 117997, Russia \\ ${ }^{5}$ Universidad Nacional de Colombia, Sede Bogotá, Observatorio Astronómico, \\ Carrera 45 \# 26-85, Bogotá, Colombia
}

\begin{abstract}
Initiation and development of a M 1.0 class flare of June 12, 2014, was observed by space and ground-based telescopes, including EUV and X-ray imaging spectroscopy by IRIS and RHESSI, and high-resolution optical imaging by $1.6 \mathrm{~m}$ New Solar Telescope (NST). Analyzing the NST data, we found small-scale loop-like structures in the region of the magnetic field Polarity Inversion Line (PIL), the emergence and interaction of which caused photospheric brightenings temporarily coinciding with hard X-ray impulses. Detailed studies of the PIL region reveal signatures of photospheric plasma downflows and dissipation of electric currents. The reconstructed magnetic field topology shows a bundle of lines connecting the PIL region with the flare ribbons which were places of chromospheric evaporation observed by IRIS. The observations suggest a scenario with the primary energy release processes located in the low atmospheric layers of the PIL, energizing the overlying large-scale magnetic structure and causing "gentle" chromospheric evaporation.
\end{abstract}

Keywords. Sun: flares, Sun: magnetic fields, Sun:photosphere, Sun: chromosphere, Sun: transition region

\section{Introduction}

Different physical processes happening during solar flares are reflected in different energy ranges (wavelengths) or spectral lines, making multiwavelength observations of solar flares very important. The multiwavelength analysis is a clue to understand flare initiation and energy release processes. The M 1.0 class solar flare, started on 2014 June 12 at around 21:00 UT in active region NOAA 12087, was observed by numerous groundbased and space-based telescopes, and was reported in several papers (Sadykov et al. 2015, Kumar et al. 2015, Sharykin et al. 2017, Sadykov et al. 2016). In this report, we summarize these findings, and describe an overall picture of the flare process.

Besides a routine coverage of the flare by GOES/XRS (Bornmann 1996), SDO/HMI (Scherrer et al. 2012) and SDO/AIA (Lemen et al. 2012) telescopes, there are unique IRIS (De Pontieu et al. 2014) observations that are available for the studied event. IRIS operated in the coarse-raster mode during the event, and obtained detailed spectra of the Mg II 2796\&2803 $\AA$, C II 1334\&1335 A, Si IV $1403 \AA$ and Fe XXI $1354.1 \AA$ lines with high spectral $(26-53 \mathrm{~m} \AA$ ), spatial $(0.33 \operatorname{arcsec})$, and temporal ( $\approx 20$ s for the loop scan) 

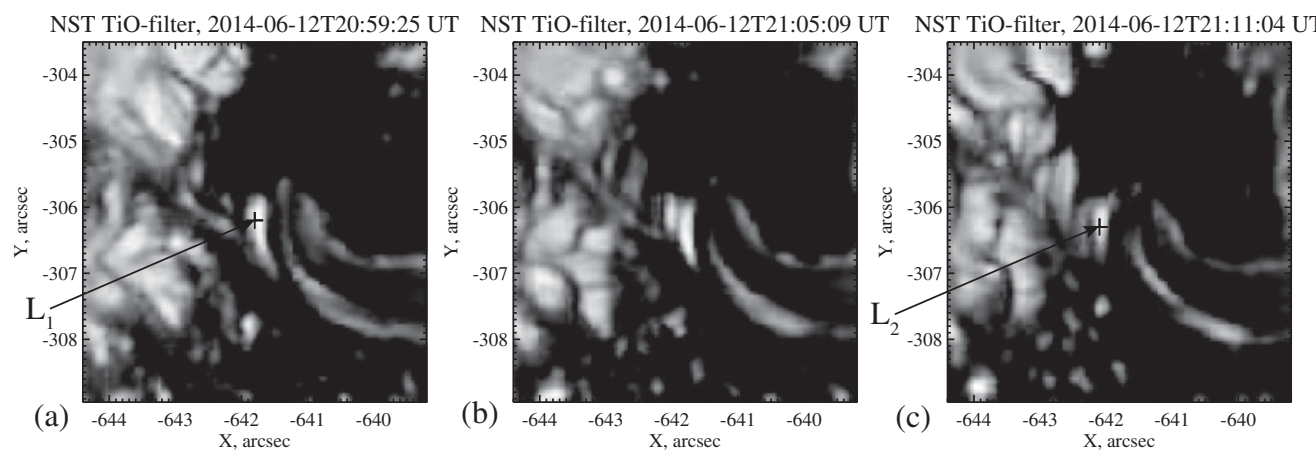

Figure 1. Emergence and interaction of photospheric small-scale loop-like structures observed with the NST BFI instrument in the region of the polarity inversion line: a) initial pre-flare loop brightening; b) emergence of a secondary loop structure; c) brightening after the loop interaction. The evolution of brightness in points $L_{1}$ and $L_{2}$ is shown in Fig. 2.

resolutions for a significant part of the flare. The high-resolution $\mathrm{H}_{\alpha}$ data from the Visible Imaging Spectrometer (VIS) and the TiO filter (7057 $\AA$ ) images from the Broadband Filter Imager (BFI) obtained with a near-diffraction-limited resolution of 0.1 arcsec at the $1.6 \mathrm{~m}$ New Solar Telescope (NST, Goode and Cao 2012) are also available. In addition, RHESSI (Lin et al. 2002) covered the entire event allowing us to analyze properties of the $\mathrm{X}$-ray radiation.

\section{Preflare Phase}

Interesting phenomena started to happen in the region even before the flare. A bright large-scale loop structure in the vicinity of the Polarity Inversion Line (PIL) in the $\delta$ type sunspot with strong magnetic field was visible in the AIA/SDO, VIS/NST and IRIS Slit-Jaw images. Later on, a strongly redshifted jet appeared near this structure (Kumar et al. 2015, Sadykov et al. 2016); it started 15 min before the flare and lasted until the flare start. Although the loop-like structure was not covered by the IRIS slit positions, the jet crossed the slit, which allowed us to study its spectra in details. In the line profiles, the jet was first reflected as an enhancement of the red wing, and later as additional strongly redshifted $(\sim 100 \mathrm{~km} / \mathrm{s})$ peak of the $\mathrm{Mg}$ II and C II spectral lines, representing the existence of two populations of plasma along the line of sight. The jet was also prominent in the $\mathrm{H}_{\alpha}$ observations, and possibly represented plasma outflow from the magnetic reconnection site located in the low atmosphere (Sharykin et al. 2017). However, the exact nature of the jet is uncovered.

\section{Flare Initiation}

At the flare start the NST BFI data showed rapid brightening of a small-scale looplike structure, $\sim 1$ arcsec long and $\sim 0.3$ arcsec wide, across the polarity inversion line (Fig. 1a). The BFI images taken with $\sim 15$-sec cadence show that the brightening started at 20:50 UT, about 10 min before the rise of the X-ray flux (Fig. 2a), and continued until 21:05 UT, when the second loop emerged (Fig. 1b) and started interacting with the first loop. The sequence of images reveals that the interaction was accompanied by a brief darkening, and formation of a single bright loop (Fig. 1c). The brightening of this loop coincides with the main hard X-ray impulse (Fig. 2b). 

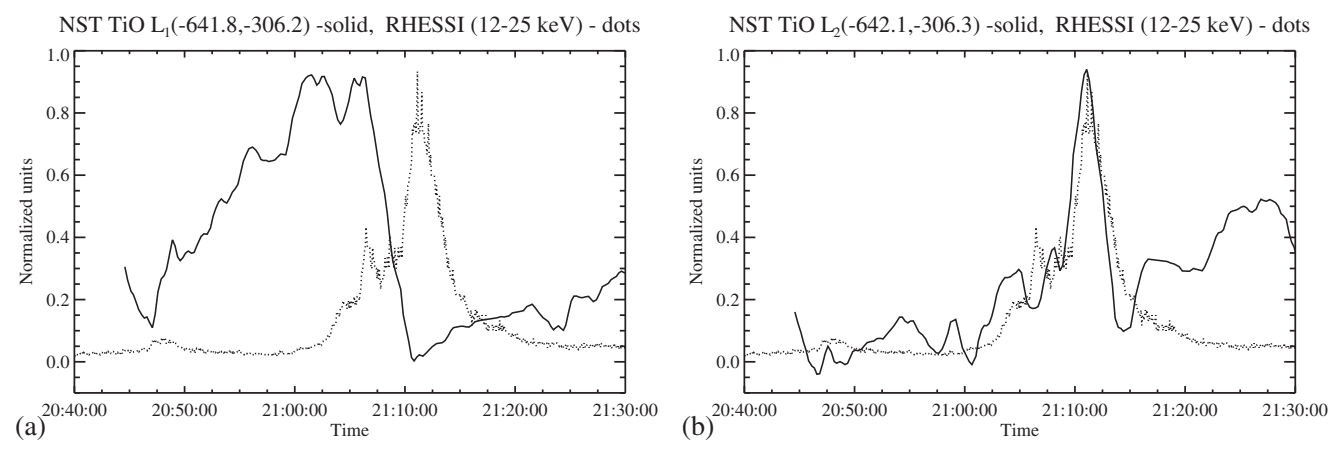

Figure 2. The evolution hard X-ray emission of the flare and the photospheric brightness of the small-scale loop structures shown in Fig. 1 for: a) the initial pre-flare brightening (point $L_{1}$ ), and b) in the region of loop interaction (point $L_{2}$ ).

The flare affected a large chromospheric region, and formed $\mathrm{H}_{\alpha}$ ribbons. One may ask a question, where the primary energy release site is located, and how the energy release was distributed across the flare region and led to the formation of the ribbons?

The most interesting feature attracting the attention in the studied flare is the emerging loop-like structures located in the vicinity of the strong PIL line (see Fig. 3). The detailed study of the region can be found in Sharykin et al. (2017), and we highlight the main results here. After the hard X-ray impulse, during the flare main phase, a flux-rope was observed as an expanding arcade in the NST TiO filter (7057 $\AA$, panels a-c of Fig. 3) and in the $\mathrm{H}_{\alpha}$ line core images, exactly matching the place of the preflare activities. The appearance of new downflows was detected with the start of the flare in the PIL site. The Non-Linear Force Free Field (NLFFF, Wheatland et al. 2000) reconstruction revealed dissipations of currents in the region possibly due to the flare energy release. The energy of the highly non-potential magnetic field was found to be comparable with the estimated flare energy, suggesting that the primary energy release was in the PIL region.

But how the energy initially generated in the PIL site was distributed across the active region? It was found that the PIL region is connected with the flare ribbons via the system of the low-lying ( $\leqslant 4.5 \mathrm{Mm}$ ) magnetic field lines (Sadykov et al. 2016). The primary energy release was in the PIL region, then the energy (in the form of heat flux or accelerated particles) could easily be distributed along the field lines to the flare ribbons (see panels b-c of Fig. 4). To validate the reconstruction, we have calculated the Quasi-Separatrix layer (QSL, Demoulin et al. 1996) usually associated with the magnetic energy dissipation. The chromospheric cross-section of the QSL corresponds to the flare ribbons and evolves in the same way (see Fig. 4), confirming the general correctness of the reconstructed magnetic field topology.

\section{Chromospheric Evaporation}

Thanks to the IRIS observations, we had an opportunity to study in details the chromospheric plasma dynamics. One of the interesting findings is the stable redshifts ( $\sim 50 \mathrm{~km} / \mathrm{s})$ across the flare region of the C II $1334 \AA$ line formed in lower transition region (Sadykov et al. 2015). Detailed study showed that the redshifts integrated over the region increase during the flare. At the same time, we detected appearance of the blueshifted Fe XXI 1354.1 $\AA$ coronal line that is formed at high temperatures (about $10 \mathrm{MK}$ ). The velocities of hot plasma upflows were also $\sim 50 \mathrm{~km} / \mathrm{s}$. Thus, despite the redshifts of the 

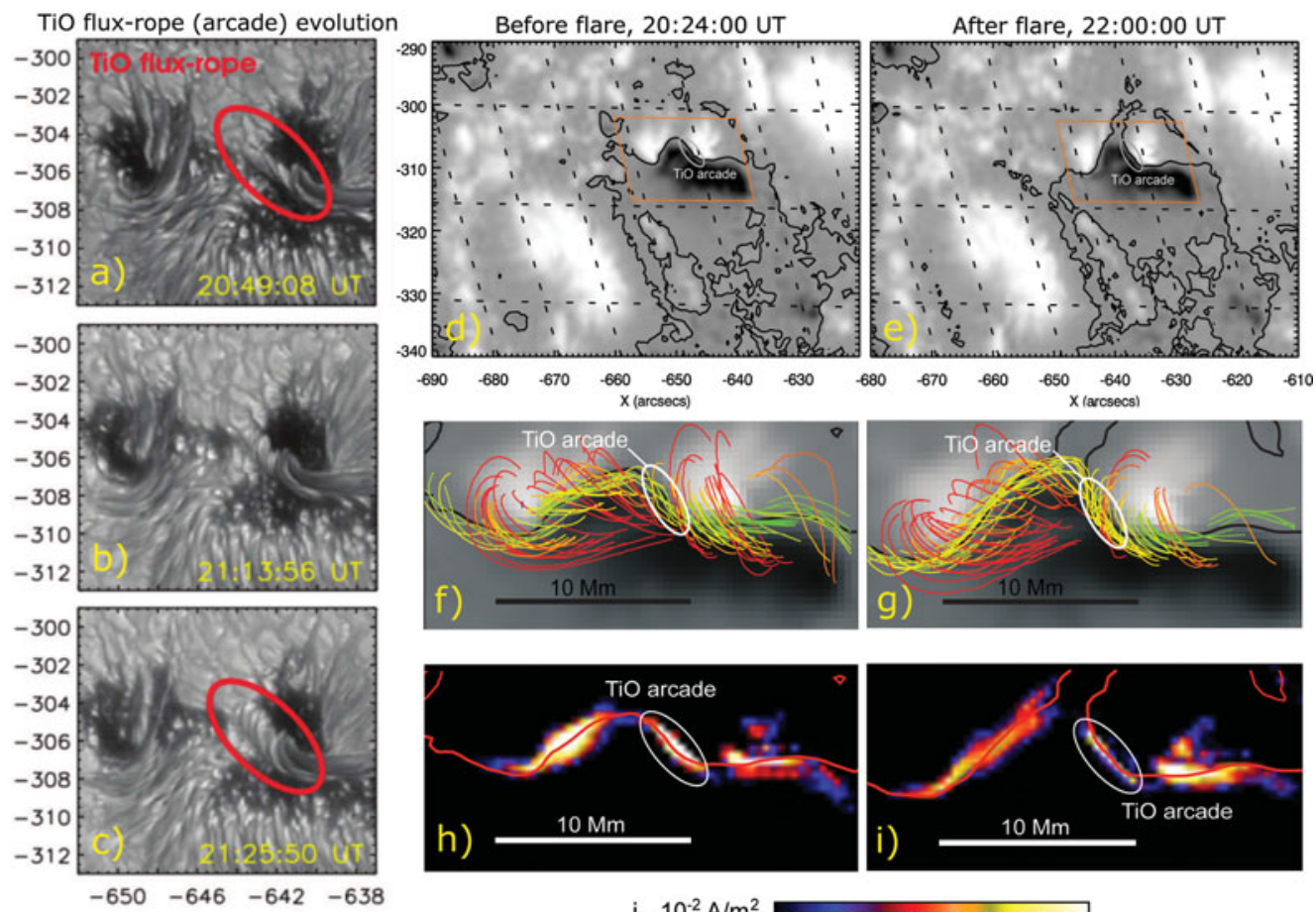

$\mathrm{j}_{\mathrm{h}}, 10^{-2} \mathrm{~A} / \mathrm{m}^{2}$

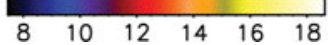

Figure 3. (a-c) Evolution of a highly twisted magnetic field structure (marked as TiO flux-rope) revealed in the NST/TiO images of the region; (d-e) The HMI LOS magnetograms in the solar disk coordinates. Orange box indicates the region selected for detailed analysis and presented on the heliographic grid in panels ( $\mathrm{f}-\mathrm{i}) ;(\mathrm{f}-\mathrm{g})$ The twisted magnetic structure elongated along the PIL. Different colors mark different heights of the field line above the photosphere (the height increases from green to red color). Black line marks the PIL; (h-i) Maps of horizontal component $\mathrm{j}_{h}$ of electric current density, calculated using the NLFFF extrapolation. Red line marks the PIL. Panels d,f and h correspond to the selected preflare time; e,g,i - to the postflare time (Sharykin et al. 2017).

cold C II line, we classify this process as "gentle" chromospheric evaporation (Antiochos and Sturrock 1978, Fisher et al. 1985). Additional analysis of the RHESSI spectra for different time intervals led to the conclusion that the evaporation can be explained by both low flux of the high-energy particles or by the heat conduction flux.

In addition, we found delays of the Fe XXI blueshift maxima relative to the chromospheric response (Sadykov et al. 2016). According to the recent numerical simulations (Rubio da Costa et al. 2015), such delays are expected to be rather short for the observed energy deposit rates. However, we observed delays of $2 \mathrm{~min}$ or even longer. The idea of the nonequilibrium ionization, as well as non-standard conditions in the low-lying loop systems need to be explored as possible explanations for the observed delays.

\section{Conclusion}

The case study of the M1.0 class solar flare shows the importance of the high-resolution multi-instrument studies. The imaging instruments on the $1.6 \mathrm{~m}$ New Solar Telescope revealed fine photospheric structures in the magnetic field Polarity Inversion Line (PIL) of the $\delta$-type sunspot with an unprecedented sub-arcsecond resolution. It has been known since observations of Severny (1958) that solar flares are typically associated with the 

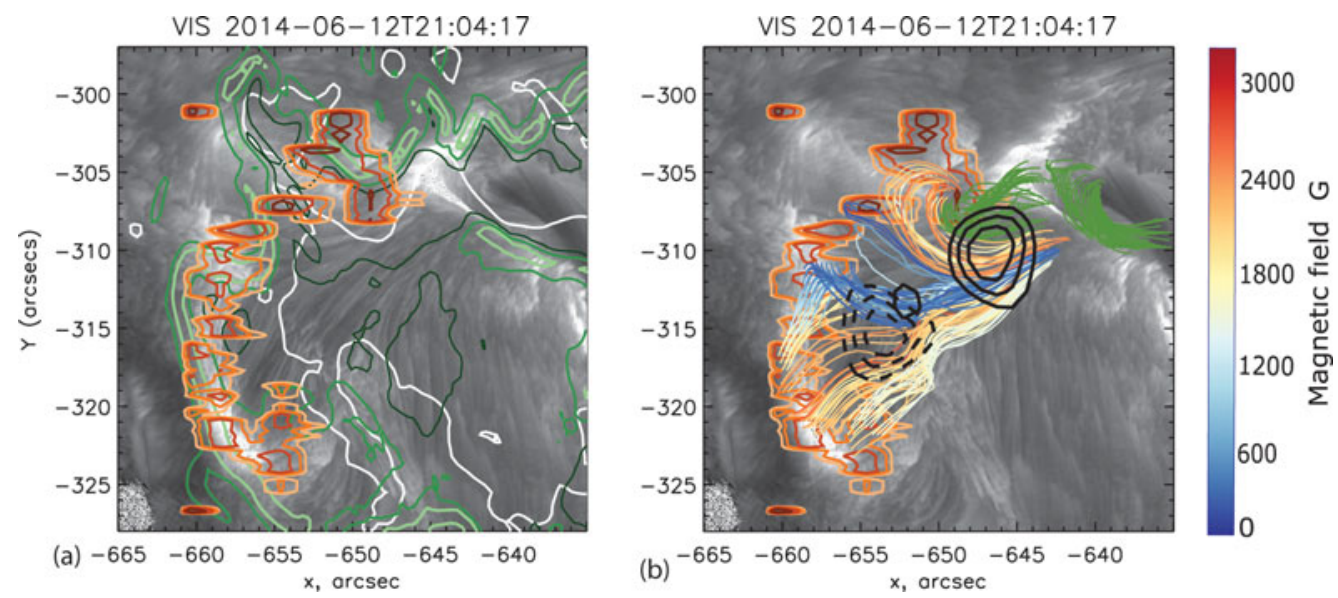

Figure 4. (a) The Fe XXI blueshift delays relative to the C II redshift maxima shown by contour lines for $30 \mathrm{~s}, 60 \mathrm{~s}, 120 \mathrm{~s}$, and $240 \mathrm{~s}$ (from light orange to dark red), and the QSL chromospheric squashing factor before the flare (for 20:58:25 UT) shown by green contours. The NST $\mathrm{H}_{\alpha}$ line core image is shown in the background in all panels. The white curve represents the PIL. (b) The reconstructed magnetic field lines with the starting footpoints in the delay regions. The line colors correspond to the magnetic field magnitude at the starting point (see the scale below the panels). The field lines corresponding to the flux rope are plotted in green. Additionally, the RHESSI $1225 \mathrm{keV}$ X-ray sources for the 21:04:00 UT21:05:40 UT (solid) and 21:05:40 UT21:06:16 UT (dashed) time periods are plotted by level contours corresponding to the $90 \%, 80 \%$, and $70 \%$ of their maxima. (Sadykov et al. 2016).

PIL of strong magnetic fields. However, the detailed PIL structure, and the physical processes that lead to the flare initiation and energy release are still enigmatic. The high-resolution data have allowed us to look, for the first time, at the fine-scale PIL dynamics that reveal small-scale brightenings associated with interacting loop-like structures, strong shearing flows and dissipation of electric current. The photospheric PIL processes were observed at the very beginning of the flare, and were coincident with the flare hard X-ray emission. The further flare development included formation of large-scale flare ribbons along the magnetic field quasi-separatrix layers (QSL), and accompanied by "gentle"-type chromospheric evaporation. Analysis of the magnetic filed topological structure found that the PIL region is connected with the flare ribbons via the system of the low-lying $(\leqslant 4.5 \mathrm{Mm})$ magnetic field lines. Thus, these observations suggest a scenario with the primary energy release processes located in the low atmospheric layers of the PIL, energizing the overlying large-scale magnetic structure, and, probably, triggering additional energy release. Further high-resolution observations in different wavelengths (energies), as well as combination of the flare spectroscopy and imaging, will provide us with more complete picture of the fine structure of solar flares, and allow us to better understand the energy release and transport mechanisms involved in the flare process.

Authors acknowledge GOES, HMI/SDO, AIA/SDO, RHESSI, IRIS and NST/BBSO observing and technical teams for the availability of the high-quality scientific data. The BBSO operation is supported by NJIT, US NSF AGS-1250818, and NASA NNX13AG14G grants, and the NST operation is partly supported by the Korea Astronomy and Space Science Institute and Seoul National University and by the strategic priority research program of CAS with Grant No. XDB09000000. The research was partially supported by the NASA Grants NNX16AP05H and NNX14AB68G. 


\section{References}

Antiochos, S. K. \& Sturrock, P. A. 1978, ApJ, 220, 1137

Bornmann, P. L., Speich, D., Hirman, J., et al. 1996, in Proc. SPIE, 2812, 291

De Pontieu, B., Title, A. M., Lemen, J. R. et al. 2014, SoPh, 289, 2733

Démoulin, P., Henoux, J. C., Priest, E. R., \& Mandrini, C. H. 1996, A\&̊A, 308, 643

Fisher, G. H., Canfield, R. C., \& McClymont, A. N. 1985, ApJ, 289, 414

Goode, P. R. \& Cao, W. 2012, ASPCS, 463, 357

Kumar, P., Yurchyshyn, V., Wang, H., \& Cho, K.-S. 2015, ApJ, 83, 809

Lemen, J. R., Title, A. M., Akin, D. J., et al. 2012, Sol. Phys., 275, 17

Lin, R. P., Dennis, B. R., Hurford, G. J. et al. 2002, Sol. Phys., 2010, 3

Rubio da Costa, F., Liu, W., Petrosian, V., \& Carlsson, M. 2015, ApJ, 813, 133

Sadykov, V. M., Vargas Dominguez, S., Kosovichev, A. G. et al. 2015, ApJ, 805, 167

Sadykov, V. M., Kosovichev, A. G., Sharykin, I. N. et al. 2016, ApJ, 828, 4

Scherrer, P. H., Schou, J., \& Bush, R. I. 2012, SoPh, 275, 207

Severnyi, A. B. 1958, Soviet Ast., 2, 310

Sharykin, I. N., Sadykov, V. M., Kosovichev, A. G. et al. 2017, ApJ, 840, 13

Wheatland, M. S., Sturrock, P. A., \& Roumeliotis, G. 2000, ApJ, 540, 1150 